\title{
Alternate primers for whole-genome SARS-CoV-2 sequencing
}

\author{
Matthew Cotten, ${ }^{1,2, *, \dagger}$ Dan Lule Bugembe, ${ }^{1}$ Pontiano Kaleebu, ${ }^{1,3}$ and \\ My V.T. Phan ${ }^{1, \neq}$
}
${ }^{1}$ MRC/UVRI \& London School of Hygiene and Tropical Medicine, 51-59 Nakiwoggo Road, Entebbe, Uganda, ${ }^{2}$ UK Medical Research Council-University of Glasgow Centre for Virus Research, Glasgow, UK and ${ }^{3}$ Uganda Virus Research Institute, Entebbe, Uganda

*Corresponding author: E-mail: matthew.cotten@lshtm.ac.uk

thttps://orcid.org/0000-0002-3361-3351

${ }^{{ }^{\prime}}$ https://orcid.org/0000-0002-6905-8513

\begin{abstract}
As the world is struggling to control the novel Severe Acute Respiratory Syndrome Coronavirus 2 (SARS-CoV-2), there is an urgency to develop effective control measures. Essential information is encoded in the virus genome sequence with accurate and complete SARS-CoV-2 sequences essential for tracking the movement and evolution of the virus and for guiding efforts to develop vaccines and antiviral drugs. While there is unprecedented SARS-CoV-2 sequencing efforts globally, approximately 19 to 43 per cent of the genomes generated monthly are gapped, reducing their information content. The current study documents the genome gap frequencies and their positions in the currently available data and provides an alternative primer set and a sequencing scheme to help improve the quality and coverage of the genomes.
\end{abstract}

Key words: SARS-CoV-2; COVID-19; primers; next generation sequencing.

\section{Introduction}

Since the first report on 30 December 2019 in Wuhan China and the WHO declaration of the pandemic on 12 March 2020, the novel Severe Acute Respiratory Syndrome Coronavirus 2 (SARSCoV-2) (Holmes and Zhang 2020) and the associated disease Coronavirus Disease 2019 (COVID-19) (Li et al. 2020; Yang et al. 2020) have continued to spread throughout the world, causing $>46$ million infections and $>1,200,000$ death globally (Gardner et al. 2020). The virus genome sequences carry important information, which can be used to interpret the virus transmission, evolution patterns and origin tracing. Furthermore, accurate and complete genomic sequences are essential for monitoring diagnostics and developing novel therapeutics and vaccines. We have seen an unprecedented amount of virus sequencing with over 130,000 complete or nearly complete genome sequences of SARS-CoV-2 now available in the GISAID database by the end of
September 2020 (Shu and McCauley 2017). Most of the sequences have been generated by next generation sequencing using targeted amplicon methods. A scan through SARS-CoV-2 genomes from GISAID with the filter 'complete genome' revealed a high frequency of gaps occurring across the genome, influencing the overall genomics quality and interpretation. Here, we describe an alternate primer scheme for whole-genome sequencing to improve the genome sequence quality and coverage.

\section{Documenting the problem}

We retrieved genomes deposited to GISAID in September 2020 (9 months into the pandemic), using the 'complete genome' filter and sorting the genomes by sequencing platforms information included in the metadata. Figure 1 illustrated the positions

(c) The Author(s) 2021. Published by Oxford University Press.

This is an Open Access article distributed under the terms of the Creative Commons Attribution License (http://creativecommons.org/licenses/by/4.0/), which permits unrestricted reuse, distribution, and reproduction in any medium, provided the original work is properly cited. 


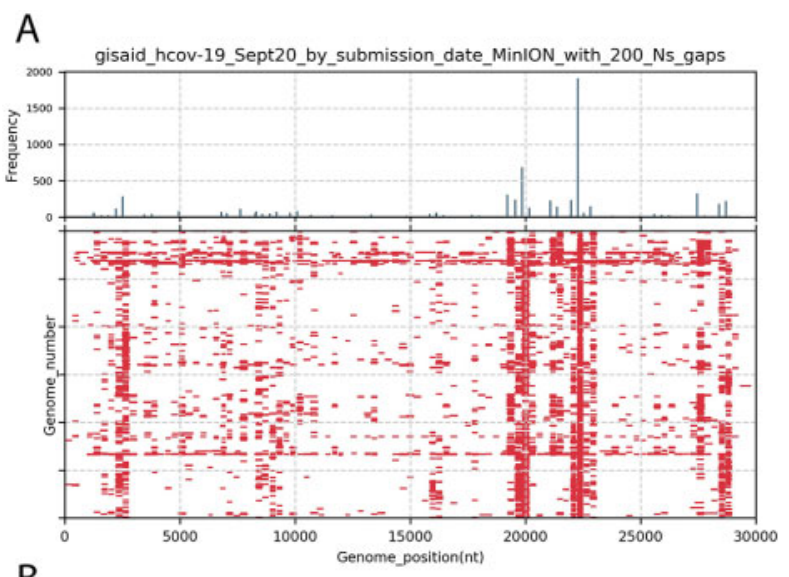

B

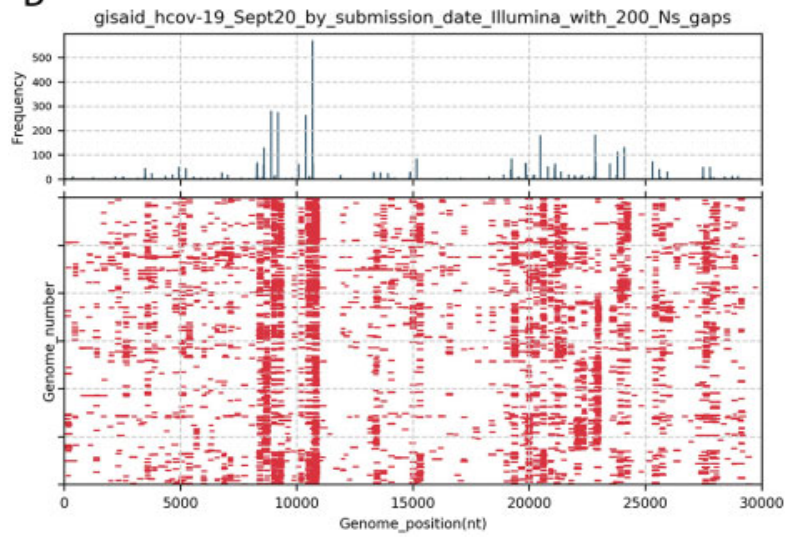

Figure 1. (updated graphics) Positions of $200 \mathrm{nt}$ gaps across SARS-CoV-2 genomes listed as complete in GISAID. Genomes deposited in September 2020 $(\mathrm{n}=38,228)$ were retrieved from GISAID, sorted by sequencing platform (MinION versus Illumina) and genomes with at least one instance of $200 \mathrm{~N}$ were collected. Panel A presents gaps in the first 3,000 MinION-generated genome sequences deposited that contained at least on $200 \mathrm{~N}$ motif. Gaps $>=200 \mathrm{nt}$ in each genome are indicated with red bars. The upper panel histogram shows the frequency (in $30 \mathrm{nt}$ bins) of gaps $>=200 \mathrm{nt}$ motifs by start position on genomes. Panel B is the same analysis of the first 3,000 Illumina-generated genome sequences in September 2020 that contained at least one $200 \mathrm{~N}$ motif.

across the $30 \mathrm{~kb}$ genome of every stretch of $200 \mathrm{Ns}$ (N200; nearly the size of an amplicon) in the first genomes deposited in September 2020 using the Illumina platform (Panel A; $N=3,000$ ) versus MinION platform (Panel $B ; N=3,000$ ). Histograms of gap frequencies across the genomes are shown for each platform. The gaps are not randomly distributed but occur with higher frequency in a subset of positions across the genome. Although genomes generated by the two platforms (Illumina and MinION) show similar problem regions (nt 8,000-11,000, and nt 19,00024,000 relative to the reference genome NC_045512), the patterns are not completely identical. Given the use of several primer amplification schemes, we suspect the gaps in coverage may be due to unexpected primer interactions, complicated sequence regions (odd composition or secondary structure), issues with primer trimming during quality control of read data, or some combinations of these factors.

The phenomenon is unlikely to be due to an isolated set of genomes as we observed similar N200 frequencies in genomes submitted from each month of the pandemic (Table 1), suggesting that gaps in coverage is a more general phenomenon. Of note, genomes generated using Ion Torrent show much lower levels of N200 (Table 1). The very low frequency of large gaps in the Ion Torrent data may be due to the use of a dedicated alternative primer set (Alessandrini et al. 2020). There have been discussions and reports on the SARS-CoV-2 genome changes due to sequencing errors as well as long gaps in the genomes due to missing amplicons from the amplicon-approach sequencing (De Maio et al. 2020; Page et al. 2020). Updates of the ARTIC primers have been presented in late March 2020 to address these issues (Quick and Loman 2020; Tyson et al. 2020). Additional reports of longer amplicon methods have been published (Eden et al. 2020; Freed et al. 2020; Gonzalez-Reiche et al. 2020), including methods to use a subset of ARTIC primers to generate longer amplicons (Itokawa et al. 2020).

However, the percentage of reported complete genomes in GISAID with 1 or more N200s continues, with 10,611 (28 per cent) of the 38,228 genomes deposited in September 2020 having 1 or more N200 gaps (Table 1), indicating the challenges remain largely unsolved.

\section{Detailed analysis of gaps}

A more focused analysis of the frequent gaps is provided in Fig. 2. The gap pattern between nt 19,000 and 24,000 (relative to the reference genome NC_045512) is shown for both MinION and Illumina sequences (first 3,000 genomes of each deposited in September 2020 with at least one $200 \mathrm{~N}$ motif). For reference, the positions of the ARTIC primers (v.1) in the region are indicated (middle panel). A histogram of gap start positions (top panel) and the individual genome gaps (bottom panel) are also shown.

The peaks of gap start positions frequently lie between forward primerL from Amplicon $\mathrm{n}$ and reverse primerR from Amplicon $\mathrm{n}-1$, for both MinION and Illumina data. Because of overlapping amplicons commonly used, if a single amplicon is missing from the sequencing library (amplicon 74 for example), the resulting gap in coverage would not be the complete amplicon 74 but would span from the $3^{\prime}$ end of the adjacent amplicon 73 (after primer and quality trimming) to the $5^{\prime}$ end of adjacent amplicon 75 (after primer and quality trimming). The calculated gaps generated by such amplicon loss have a median length of $270.5 \mathrm{nt}$, which is close to the the observed median gap length in the MinION data (258nt) or Illumina data (262 nt) from September 2020. This arrangement is outlined in the Supplementary Fig. S1, Panel A.

\section{Alternate primers as a potential solution to avoid gapped genomes}

We explored an alternate set of amplification primers (termed the Entebbe primers) designed using methods we had previously used for MERS-CoV (Cotten et al. 2013), Norovirus (Cotten et al. 2014), RSV (Agoti et al. 2015) and Yellow Fever virus (Phan et al. 2019). Important for the design were the amplicon size and the primer placement. For their implementation, the use of primers for the reverse transcription step, and the multiplexing of the amplicons in two staggered sets were important for the PCR. Our experience had suggested an optimum amplicon size of around $1500 \mathrm{bp}$. The larger amplicons reduced the total primers content of the reactions but still allowed high reverse transcription efficiency (which, in our hands, declined beyond $1500 \mathrm{nt}$ ). Here we describe primers designed for whole-genome sequencing of SARS-CoV-2, as well as sharing the detailed laboratory methods that we used for reverse transcription, PCR 
Table 1. Frequency of SARS-CoV-2 genomes with 1 or more $200 \mathrm{nt}$ gaps (N200) by month and by sequencing platform.

\begin{tabular}{|c|c|c|c|c|c|c|c|c|c|c|c|}
\hline Deposition period & $\begin{array}{l}\text { Complete } \\
\text { genomes }^{\mathrm{a}}\end{array}$ & $\begin{array}{l}\text { Genomes } \\
\text { with } 1 \text { or } \\
\text { more } \\
\text { N200 }\end{array}$ & $\begin{array}{l}\% \text {. with } \\
1 \text { or } \\
\text { more } \\
\text { N200 }\end{array}$ & $\begin{array}{l}\text { Illumina } \\
\text { total }^{\mathrm{d}}\end{array}$ & $\begin{array}{l}\text { Illumina \% } \\
\text { with } \\
\text { N200 }\end{array}$ & $\begin{array}{l}\text { MinION } \\
\text { total }^{\mathrm{f}}\end{array}$ & $\begin{array}{l}\text { MinION \% } \\
\text { with } \\
\text { N200" }\end{array}$ & $\begin{array}{l}\text { Ion } \\
\text { Torrent } \\
\text { total }^{\text {h }}\end{array}$ & $\begin{array}{l}\text { Ion } \\
\text { Torrent \% } \\
\text { with } \\
\text { N200 }\end{array}$ & $\begin{array}{l}\text { Method } \\
\text { unclear }^{j}\end{array}$ & $\begin{array}{l}\text { Method } \\
\text { unclear } \% \\
\text { with } \mathrm{N}^{200^{\mathrm{k}}}\end{array}$ \\
\hline 1-31 January 2020 & 54 & 1 & 2 & 9 & 0 & 2 & 0 & 0 & 0 & 43 & 2 \\
\hline 1-29 February 2020 & 126 & 2 & 2 & 44 & 0 & 9 & 0 & 5 & 0 & 65 & 4 \\
\hline 1-31 March 2020 & 2,872 & 559 & 19 & 1,518 & 16 & 548 & 32 & 35 & 6 & 771 & 18 \\
\hline 1-30 April 2020 & 12,411 & 3,745 & 30 & 4,970 & 38 & 1,286 & 27 & 264 & 0 & 5,424 & 26 \\
\hline 1-31 May 2020 & 19,787 & 8,606 & 43 & 8,634 & 52 & 2,634 & 30 & 529 & 0 & 7,990 & 42 \\
\hline 1-30 June 2020 & 21,665 & 8,723 & 40 & 7,043 & 36 & 3,844 & 35 & 629 & 2 & 10,149 & 47 \\
\hline 1-31 July 2020 & 17,986 & 4,834 & 27 & 4,965 & 23 & 1,585 & 33 & 471 & 2 & 10,965 & 29 \\
\hline 1-31 August 2020 & 17,276 & 4005 & 23 & 11,074 & 22 & 2,270 & 26 & 486 & 0 & 3,446 & 28 \\
\hline 1-30 September 2020 & 38,227 & 10,611 & 28 & 22,740 & 23 & 7,973 & 44 & 580 & 1 & 6,934 & 28 \\
\hline
\end{tabular}

${ }^{a}$ Number of genomes with the annotation 'complete' retrieved from GISAID (https://www.gisaid.org/).

${ }^{\mathrm{b}} \mathrm{Genomes}$ were sorted by the presence or absence of the sequence N200.

'((The number of genomes with at least one N200)/total number of genomes)* 100 .

dNumber of genomes in GISAID for this period generated using any of the Illumina methods as noted in the GISAID 'Sequencing technology metadata'.

${ }^{e}$ ((The number of Illumina genomes with at least one N200)/total number of genomes)*100.

f,g Number of genomes in GISAID for this period generated using any of the MinION methods as noted in the GISAID 'Sequencing technology metadata' and their percentage.

${ }_{\mathrm{h}, \mathrm{i}}$ Number of genomes in GISAID for this period generated using any of the Ion Torrent methods as noted in the GISAID 'Sequencing technology metadata' and their percentage.

${ }_{j, k}$ Number of genomes in GISAID for this period generated using unclear methods as noted in the GISAID 'Sequencing technology metadata' and their percentage.

\section{A MinION data}

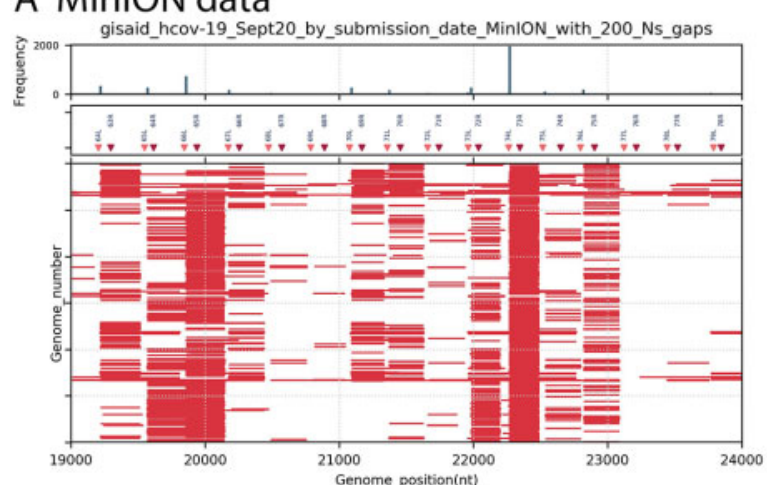

\section{B Illumina data}

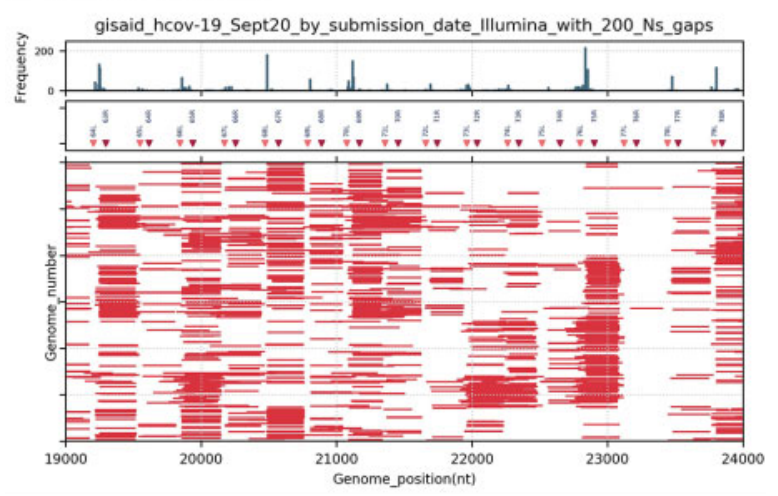

Figure 2. Positions of $200 \mathrm{nt}$ gaps across SARS-CoV-2 genomes stratified by MinION or Illumina, in region nt 19,000 to 24,000 . Genomes deposited in September 2020 as 'complete' were retrieved from GISAID, sorted by sequencing platform and by the presence of at least one N200 motif. For clarity, only the first 3,000 genomes in each set were plotted. Similar to Figure 1, gaps $>=200 \mathrm{nt}$ in each genome are indicated with red bars. The upper panel histogram shows the frequency (in $30 \mathrm{nt}$ bins) of gaps $>=200 \mathrm{nt}$ motifs by start position on genome, the middle panel plots the positions of ARTIC v.1 primers in the region (pink = forward 'left' primers, red = reverse 'right' primers). Panel A: MinION-derived genome sequences, Panel B: Illumina-derived genome sequences. amplification and MinION library preparation to successfully sequence the SARS-CoV-2 genome.

Briefly, the primer design (Fig. 3A) started with the set of complete SARS-CoV-2 genome sequences available in the GISAID database on 22 June $2020(\mathrm{~N}=21,687)$. Spaces and disruptive characters were removed from the sequence IDs and the sequences were further screened to remove genomes containing gaps of $6 \mathrm{Ns}$ or more, resulting in 17,220 clean genome sequences. Next, all sequences were sliced into $33 \mathrm{nt}$ strings (33mers), with a $1 \mathrm{nt}$ step and 606,389 unique 33mers were generated. The frequency of each 33 mer was counted to identify highly conserved 33mers. This counting method avoids the multiple sequence alignment step commonly used in primer design and becomes prohibitive with large and or diverse genome sets. This alignment-free approach allowed us to use all suitable genome sequences of interest rather than a set that could be conveniently aligned. Finally, primer-like 33mers sequences were generated by trimming the sequences to a calculated desired melting temperature and removing any primers greater than $26 \mathrm{nt}$.

In the second step, we defined forward and reverse primer target regions (bins) for the amplicons. For SARS-CoV-2, we selected twenty amplicons with an overlap of $300 \mathrm{nt}$, regularly spaced across the SARS-CoV-2 genome sequence (Fig. 3B). We then selected the top conserved primer sequences (the highest frequency primers) mapping in the $5^{\prime}$ or $3^{\prime} 185 \mathrm{nt}$ of each amplicon. For security, the two highest frequency primers per bin were selected for the SARS-CoV-2 sequence, this provided some insurance against primer failure either due to target evolution or unexpected secondary structure. The binning and primer target locations for the final set of primers are shown in Fig. 2 and the final calculated amplicon lengths were 1,495-2,093 nt.

The reverse transcription, PCR amplification and library protocols were modified to accommodate the new primers. Important changes to note are the following. Reverse transcription was performed using the reverse primers and reverse transcription at $42^{\circ} \mathrm{C}$. The PCR cycling conditions (using Phusion 
A
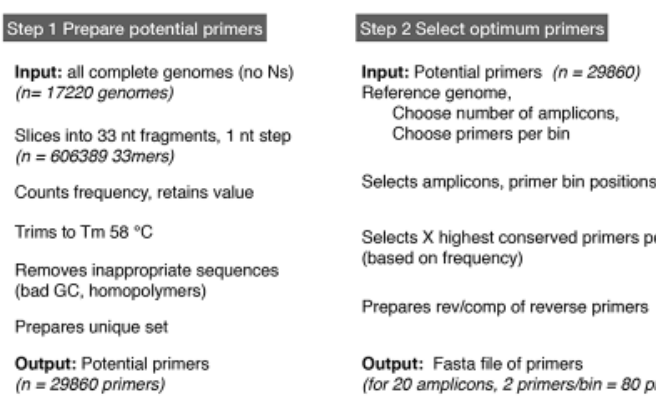

Selects $X$ highest conserved primers per bin (based on frequency)

Prepares rev/comp of reverse primers

Output: Fasta file of primers

(for 20 amplicons, 2 primers/bin $=80$ primers)

B

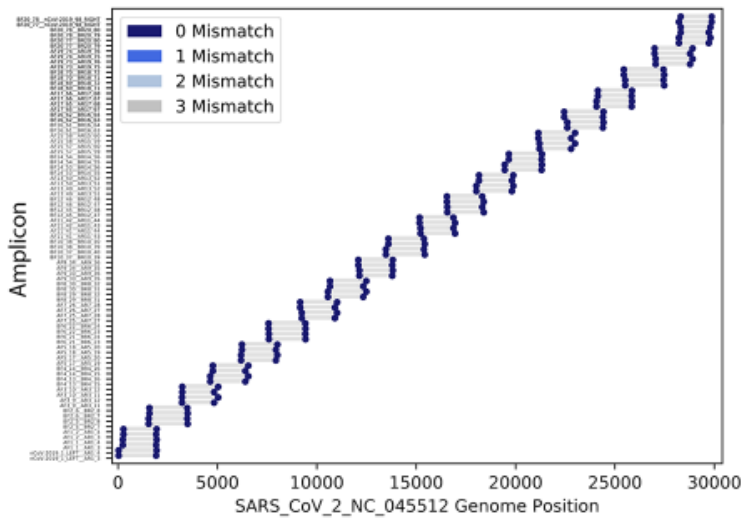

Figure 3. Primer design and amplicon layout. Panel A: The two main steps involved in primers generation and selection are shown. Panel B: The layout of the twenty amplicons across the SARS-CoV-2 genome is shown in lower panel. The blue markers indicate target positions in the SARS-CoV-2 genome (NC_045512 used here), the grey bars indicate the resulting amplicon.

enzyme) were adjusted for the new $\mathrm{T}_{\mathrm{m}} \mathrm{s}$ and an increased elongation time required for the longer PCR products. Finally, the library purification steps were adjusted to recover longer PCR and library products. A detailed step-by-step protocol is provided in the Supplementary material.

\section{Testing the performance of primers to sequence SARS-CoV-2 using MinION}

We tested the Entebbe primers performance for sequencing SARS-CoV-2 from nucleic acid extracted from positive samples. The amplicon sizes and genome coverage are summarised in Fig. 4. In particular, panels A and B (Fig. 4) illustrate the amplicon products after the reverse transcription and PCR amplification with the expected sizes of 1,400-2,000 bp. These amplicons were then pooled and used for library preparation using the MinION sequencing kits SQK-LSK109. Final libraries were quantified and sequenced using a MinION Flow Cell (R9.4.1). The resulting read data, after quality and primer and adapter trimming, were then mapped to the SARS-CoV-2 Wuhan1 reference genome NC_045512 (Fig. 4, panels C and D) to document sequence coverage across the genome. The twenty individual amplicons are detected in the coverage pattern with small peaks appearing where amplicons overlap. The coverage is consistent across the genome with no missing amplicons and the data were readily assembled into good coverage full genomes. Initial experiments showed that amplicons 2 (spanning nt 2,400) and 16 (spanning nt 23,500) had reduced yields (Fig. 4, panel C). The primer mixes were subsequently adjusted to increase concentrations of amplicon 1 and 16 primers for reverse transcription and PCR (see detailed protocol in Supplementary materials), this improved yields relative to the other amplicons (Fig. 4, panel D).

A set of SARS-CoV-2 clinical samples were tested with the Entebbe primers/protocol. Respiratory swab samples from 111 PCR confirmed cases of SARS-CoV-2 infection were processed for reverse transcription/PCR using the Entebbe primers and protocol as described in Supplementary materials. If sufficient amplicon DNA was generated after PCR, MinION libraries were prepared, samples were sequenced on the MinION flowcells and the resulting data were assembled into genomes. Figure $5 \mathrm{~A}$ shows the results of this validation test. Complete genomes (fraction genome $=1$ ) were obtained from samples up to Ct 35 ; nineteen samples failed to yield sufficient amplicon DNA after PCR stage (Fig. 5A, red markers) and five samples yielded genomes with gaps $>842 \mathrm{nt}$. The PCR failures and the gapped genomes were not strictly associated with higher Cts and their distribution pattern is similar to the overall Ct distribution pattern across the set of samples (Fig. 5, panel B) suggesting other factors such as sample quality, extraction method, storage, might be more critical than $\mathrm{Ct}$ in determining sequencing success, at least in samples within the Ct range tested (up to Ct 37).

\section{Conclusions}

Given the urgency of controlling the SARS-CoV-2 pandemic and the importance of having good quality SARS-CoV-2 genomes, we are providing these alternative primers (the Entebbe primers) with detailed step-by-step laboratory protocols to the community with the hope that they benefit from the new design. The costs and efforts of sequencing SARS-CoV-2 in the large case numbers that are currently being seen are substantial and if these new primers result in a higher proportion of gapless genomes, this will provide added value and will increase the utility of the resulting data.

\section{Supplementary data}

Supplementary data are available at Virus Evolution online.

\section{Acknowledgements}

We thank all global SARS-CoV-2 sequencing groups for their open and rapid sharing of sequence data and GISAID for providing an effective platform for making these data available. We are grateful to the Oxford Nanopore Technologies and the ARTIC Network for their support with protocols and analysis software. We thank the Central Public Health Laboratory, Uganda especially Isaac Ssewanyana, Patrick Semanda, Susan N. Nabadda for their support with SARSCoV-2 samples.

\section{Funding}

We acknowledge the support of the Uganda Ministry of Health and its COVID-19 Scientific Advisory Committee, the National COVID-19 Task Force, and the staff of the Emerging and Remerging Infections Department of the Uganda Virus Research Institute, and the US Centers for Disease Control and Prevention. The SARS-CoV-2 diagnostic and sequencing award is jointly funded by the UK MRC and the UK DFID under the MRC-DFID Concordat agreement (NC_PC_19060) and is also part of the European and Developing Countries 
A

B
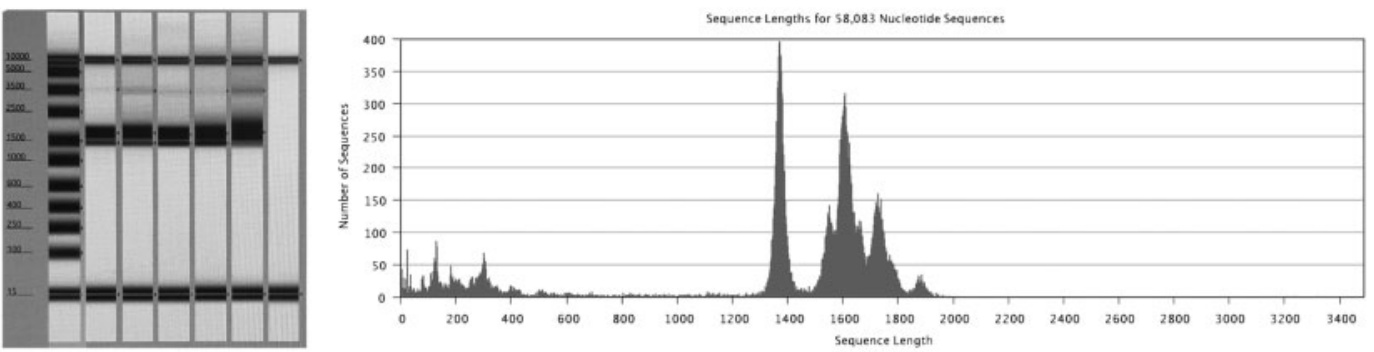

C

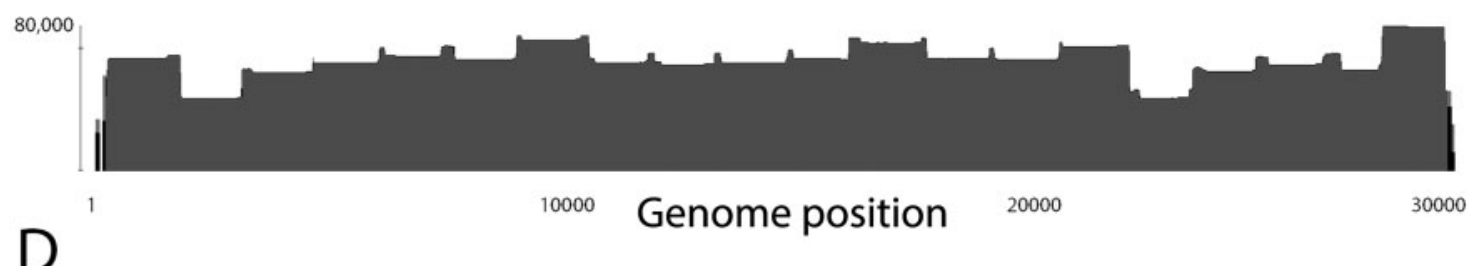

$\mathrm{D}$

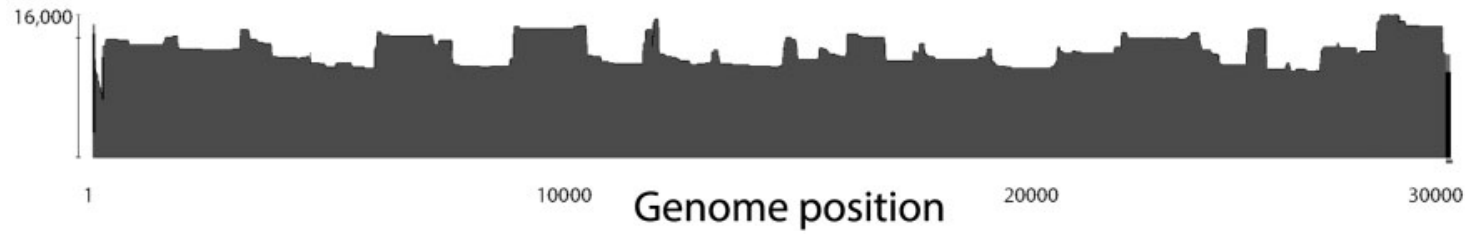

Figure 4. Testing the primer performance. Panel A: PCR product size after pooling of reaction A and B. Expected sizes of amplicons are from 1,500 bp to 2,093 bp before primer trimming. Panel B: MinION reads after quality control, primer, adapter trimming. Panel C: Reads mapped to SARS-CoV-2 reference genome, before amplicon 2 and 16 primer boosting. Panel D: Reads mapped to SARS-CoV-2 reference genome, after amplicon 2 and 16 primer boosting.

A

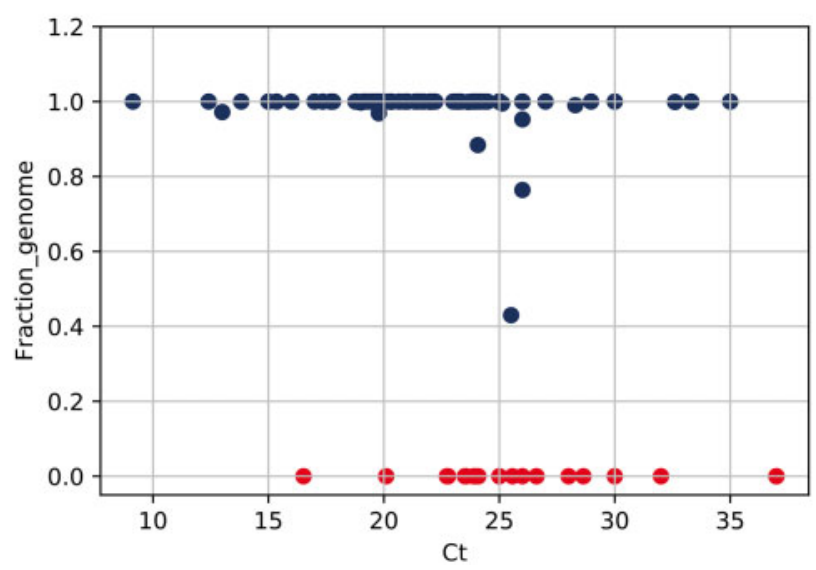

B

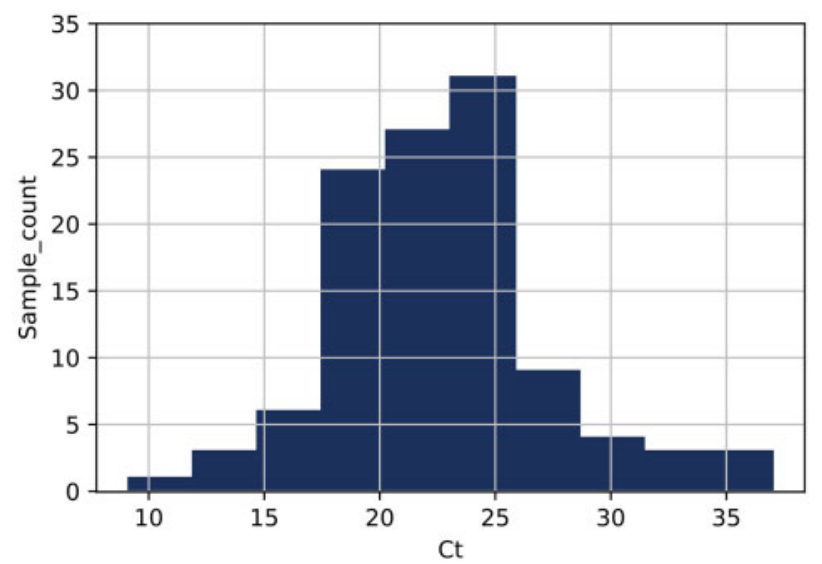

Figure 5. Validation of Entebbe primers. Panel A plots the genome yield (fraction of complete genome) as a function of sample Ct. Fraction genome was calculated by number of nonN nucleotides/29,303 (the length, in nt, of NC_045512 reference genome). Each marker represents a sample, red markers indicate 19 samples that failed to yield sufficient DNA for library, 93 that proceeded to library preparation and sequencing (dark blue markers). Panel B is a histogram of the distribution of the 118 sample Cts.

Clinical Trials Partnership 2 program supported by the European Union. The diagnostics were also supported by the UK Research and Innovation/MRC, the Global Fund, the Government of Uganda, the Islamic Development Bank, the World Health Organization, GAVI, the US Centers for Disease Control and Prevention, and the Jack Ma Foundation, among others. M.V.T.P. was supported by a Marie Sklodowska-Curie
Individual Fellowship, funded by European Union's Horizon 2020 research and innovation programme (799417). The Uganda Medical Informatics Centre high performance computer was supported by the UK MRC (MC_EX_MR/L016273/1) to P.K. The study is supported by a Wellcome Epidemic Preparedness-Coronavirus grant, jointly funded by the Wellcome Trust and UK DFID (220977/Z/20/Z) awarded to 
M.C. This study was approved by the UVRI Research and Ethics Committee (approval no. 00001354, study reference no. GC/127/20/04/771).

Conflict of interest: None declared.

\section{References}

Agoti, C. N. et al. (2015) 'Local Evolutionary Patterns of Human Respiratory Syncytial Virus Derived from Whole-Genome Sequencing', Journal of Virology, 89: 3444-54.

Alessandrini, F. et al. (2020) 'Evaluation of the Ion Ampliseq SARS-CoV-2 Research Panel by Massive Parallel Sequencing', Genes, 11: 929.

Cotten, M. et al. (2013) 'Full-Genome Deep Sequencing and Phylogenetic Analysis of Novel Human Betacoronavirus', Emerging Infectious Diseases, 19: 736-42B.

_ et al. (2014) 'Deep Sequencing of Norovirus Genomes Defines Evolutionary Patterns in an Urban Tropical Setting', Journal of Virology, 88: 11056-69.

De Maio, N. et al. 2020. 'Issues with SARS-CoV-2 Sequencing Data.' <https://virological.org/t/issues-with-sars-cov-2-se quencing-data/473> accessed 26 Jan 2021.

Eden, J.-S., for the 2019-nCoV Study Group. et al. (2020) 'An Emergent Clade of SARS-CoV-2 Linked to Returned Travellers from Iran', Virus Evolution, 6: veaa027.

Freed, N. E. et al. (2020) 'Rapid and Inexpensive Whole-Genome Sequencing of SARS-CoV-2 Using $1200 \mathrm{bp}$ Tiled Amplicons and Oxford Nanopore Rapid Barcoding', Biology Methods and Protocols, 5: bpaa014.

Gardner, L. et al. (2020). 'Coronavirus COVID-19 Global Cases by Johns Hopkins CSSE.' <https://www.arcgis.com/apps/ops dashboard/index.html\#/bda7594740fd40299423467b48e9ecf6> accessed 26 Jan 2021.
Gonzalez-Reiche, A. S. et al. (2020) 'Introductions and Early Spread of SARS-CoV-2 in the New York City Area', Science, 369, 297-301.

Holmes, E. C., and Zhang, Y.-Z. (2020) 'Initial Genome Release of Novel Coronavirus'. <http://virological.org/t/initial-genome-re lease-of-novel-coronavirus/319> accessed 26 Jan 2021)

Itokawa, K. et al. (2020) 'Disentangling Primer Interactions Improves SARS-CoV-2 Genome Sequencing by Multiplex Tiling PCR', Plos One, 15: e0239403.

Li, Q. et al. (2020) 'Early Transmission Dynamics in Wuhan, China, of Novel Coronavirus-Infected Pneumonia', New England Journal of Medicine, 382: 1199-207.

Page, A. J. et al. 2020. 'Large Scale Sequencing of SARS-CoV-2 Genomes from One Region Allows Detailed Epidemiology and Enables Local Outbreak Management', Epidemiology. https:// doi.org/10.1101/2020.09.28.20201475.

Phan, M. V. et al. (2019) 'Genomic Sequence of Yellow Fever Virus from a Dutch Traveller Returning from the Gambia-Senegal Region, The Netherlands, November 2018', Eurosurveillance, 24: 1800684.

Quick, J., and Loman, N. 2020. 'NCoV-2019 Version 3 Amplicon Release.' <https://community.artic.network/t/ncov-2019-ver sion-3-amplicon-release/19> accessed 26 Jan 2021.

Shu, Y., and McCauley, J. (2017) 'GISAID: Global Initiative on Sharing All Influenza Data - from Vision to Reality', Eurosurveillance, 22: 30494.

Tyson, J. R. et al. 2020. 'Improvements to the ARTIC Multiplex PCR Method for SARS-CoV-2 Genome Sequencing Using Nanopore', Genomics. https://doi.org/10.1101/2020.09.04.283077.

Yang, X. et al. (2020) 'Clinical Course and Outcomes of Critically Ill Patients with SARS-CoV-2 Pneumonia in Wuhan, China: A Single-Centered, Retrospective, Observational Study', The Lancet Respiratory Medicine, 8: 475-81. 